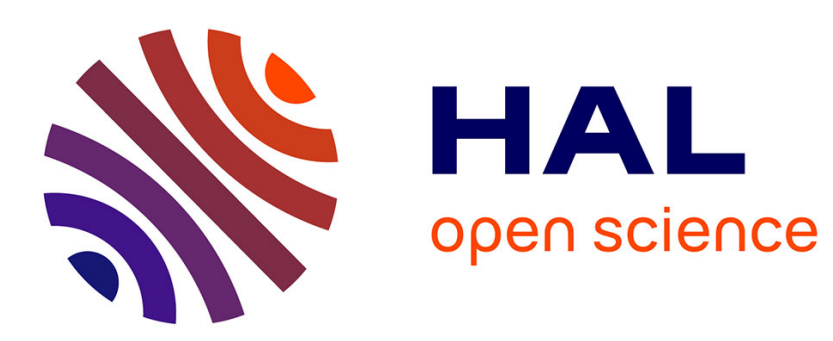

\title{
Integrating stochastic discrete constraints in MPC. Application to Home Energy Management System
}

Jesse James Arthur Prince Agbodjan, Pierre Haessig, Romain Bourdais, Hervé Guéguen

\section{- To cite this version:}

Jesse James Arthur Prince Agbodjan, Pierre Haessig, Romain Bourdais, Hervé Guéguen. Integrating stochastic discrete constraints in MPC. Application to Home Energy Management System. IFAC Journal of Systems and Control, 2021, 17, pp.100168. 10.1016/j.ifacsc.2021.100168 . hal-03367535

\section{HAL Id: hal-03367535 \\ https://hal.science/hal-03367535}

Submitted on 24 Nov 2021

HAL is a multi-disciplinary open access archive for the deposit and dissemination of scientific research documents, whether they are published or not. The documents may come from teaching and research institutions in France or abroad, or from public or private research centers.
L'archive ouverte pluridisciplinaire HAL, est destinée au dépôt et à la diffusion de documents scientifiques de niveau recherche, publiés ou non, émanant des établissements d'enseignement et de recherche français ou étrangers, des laboratoires publics ou privés. 


\title{
Integrating stochastic discrete constraints in MPC. Application to Home Energy Management System
}

\author{
Jesse-James PRINCE AGBODJAN * Pierre HAESSIG* \\ Romain BOURDAIS * Hervé GUEGUEN * \\ * IETR (Institut d'Electronique et des Technologies du numéRique), \\ CentraleSupelec Rennes, France, (e-mails : \\ $\{$ jesse-james.prince-agbodjan, pierre.haessig, romain.bourdais, \\ herve.gueguen\}@centralesupelec.fr)
}

\begin{abstract}
In this paper, in a Model Predictive Control problem, we tackle the integration of uncertain constraints that belong to a discrete set. We propose a controller that offers a solution to reformulate the discrete stochastic constraints to avoid the exponential growth of scenario tree experienced by existing controllers. The proposed controller's efficiency is shown by benchmarking and comparing it with the Multistage Stochastic Programming algorithm in a context of Home Energy Management System. The comparison is made based on simulations using real-life data to manage the energy flow within a building connected to the electricity feeder, which random character involves its availability and nonavailability.
\end{abstract}

Keywords: Model Predictive Control; Open Loop Feedback Control; Multi Stage Stochastic Programming, Grid outage, Home Energy Management System.

\section{INTRODUCTION}

Optimization-based controllers, especially Model Predictive Control (MPC) (Camacho and Alba, 2007, Borrelli 2017, James B. Rawlings, 2017), provides a practical solution that easily handles physical and operational constraints which have to be considered. However, constraints can be subject to uncertainties and disturbances, which can severely deteriorate the system's performance. This is the case when the uncertainty does not varies within a continuous space as with discrete constraints that model radical changes. Grid outages which we focus on in this paper is one example.

Two families of MPC can address uncertainty: Robust MPC and Stochastic MPC. An extensive review of both methods are proposed in (Mayne, 2014, 2016). When no other information is available, but the uncertainty is known to lie in a given set, explicitly assessing the worstcase uncertainty leads to robust optimization (Ben-Tal et al. 2009. Bram et al. 2015) in general and Robust MPC (RMPC) techniques (Bemporad and Morari, 1999 Raković, 2015) in particular. RMPC has proven its worth in many applications, but its conservativeness can be a downside for applications that interest us.

Less conservative approaches exploit the knowledge of the probability distribution of uncertain variables, which leads to stochastic MPC (SPMC). There are two main approaches to SMPC. There are two main approaches to SMPC. When the uncertain variables vary slightly around some mean value, the first approach is based on Chance Constrained optimization (Charnes et al., 1958 Prékopa, 1970, Henrion, 2007; Nasir et al., 2019), which provides explicit probabilistic guarantees on the feasibility of optimal solutions. Although very attractive, chance constrained problems can be challenging to solve in the presence of discrete random variables (r.vs.) (Adam et al. 2018; Geng and Xie, 2019).

The second approach that is better suited to discrete uncertainties is the Multistage Stochastic Programming (MSP) based on a scenario tree. When the size of the prediction horizon and the number of elements in the random variables' sample space lead to an exponential growth of the scenario tree, a simple strategy to cut this growth is to consider that the uncertainty becomes constant after the "robust horizon". This simplification is allowed by the receding horizon nature of the strategy; Modeling the far future very accurately is not critical because all the control inputs will be recomputed at the next sampling time anyway (Lucia et al. 2014). Another method that helps to avoid dealing with the exponential growth of the scenario is the Stochastic Dual Dynamic Programming (SDDP) (Pereira and Pinto, 1991) that reformulate the initial problem in dynamic programming and solve it through bender's cut method as applied in energy management by Pacaud et al. (2018).

In this paper, we propose a new method to integrate discrete stochastic constraints within a MPC framework. We call it Stochastic Discrete Constraint Model Predictive Control (SDCMPC). It is based on Open Loop Feedback Control (OLFC) (Dreyfus, 1964, Papageorgiou, 1988, Bertsekas, 2005) with a relaxation of stochastic constraints. In our method, the size of the online optimization problem grows linearly with the prediction horizon. 
In our recent work (Prince Agbodjan et al. 2019), we were interested in how to integrate deterministic radical changes within a predictive controller and therefore make it reactive to the apparition of these changes while in (Prince Agbodjan et al., 2020 the integration and anticipation of stochastic radical changes have been addressed in a study case. This paper offers a unified framework to generalise both the problem and the solution provided. The latter is used to design and bench-mark a controller that is compared to a state of the art's controller. Specifically, we extend the previous works in the following ways:

1. An explicit formalisation of the problem considered and the proposed method to solve this problem are included;

2. The resulting controller is extensively compared to the Multistage Stochastic Programming controller through Monte Carlo simulations. The latter considers disparate profiles of electrical grid availability to analyse the controllers in different situations;

The remainder of the paper is structured as follows. Section 2 presents the family of dynamical systems that we consider. In Section 3, we formulate the decision problem that we are interested in and present an existing solution based on MSP. Section 4 describes the new controller which we apply to a Home Energy Management System presented in section 5. Discussions and experimental results based on real condition data are shown in section 6 and the conclusion with some ideas of how this present work could be extended follows in section 7 .

\section{SYSTEM DESCRIPTION}

Let us consider the state-space representation of a discretetime linear system given by:

$$
x(k+1)=A x(k)+B u(k)
$$

where $x(k) \in \mathbb{R}^{n_{x}}$ and $u(k) \in \mathbb{R}^{n_{u}}$ are the model state and control vectors at the $k^{\text {th }}$ sampling instant.

The state constraints are given by:

$$
x(k) \in \mathcal{X} .
$$

Two groups of control constraints are considered:

- $n_{d}$ deterministic linear constraints that can be aggregated as:

$$
D_{d} u(k) \leq d
$$

where $D_{d} \in \mathbb{R}^{n_{d} \times n_{u}}$, and $d \in \mathbb{R}^{n_{d}}$ a column vector.

- $n_{s}$ stochastic linear inequality constraints. Each of them has the following expression:

$$
e_{i}^{T} u(k) \leq \boldsymbol{\xi}_{i}(k),
$$

where $i \in\left\{1, \ldots, n_{s}\right\}$ indexes the $i^{t h}$ stochastic inequality, $e_{i} \in \mathbb{R}^{n_{u}}$ and $\boldsymbol{\xi}_{i}$ is the associated random variable. Each $\boldsymbol{\xi}_{i}$ takes its value in a finite discrete set

$$
\Xi_{i}=\left\{\xi_{1, i}, \xi_{2, i}, \ldots \xi_{N_{i}, i}\right\},
$$

where each $\xi_{\cdot, i} \in \mathbb{R}, N_{i}=\left|\Xi_{i}\right|$, and without loss of generality the elements of the set $\Xi_{i}$ are ordered as follows

$$
\xi_{1, i}<\xi_{2, i}<\ldots<\xi_{N_{i}, i} .
$$

Note that for the rest of the paper, the following convention is adopted: a r.v. will be denoted by a bold letter, and its realization will be indicated by a regular letter.
We make the following assumption,

A.1. For all $i=1, \ldots, n_{s}$, for all realizations of $\boldsymbol{\xi}_{i}(k)$ and for all $x \in \mathcal{X}$, it exists $u(k)$ that satisfies (3) and (4) such that $x(k+1) \in \mathcal{X}$

\section{PROBLEM STATEMENT}

\subsection{MPC formulation}

To control the system, we use an MPC framework. Thus, at each instant $k$, a sequence of predicted control values is computed by minimizing a performance cost over the prediction horizon $\mathrm{H}$. Then, only the first element of the control sequence is applied to the system.

The problem is defined as follows

$$
\begin{aligned}
& \min _{u} J(k)=\sum_{h=0}^{H-1} j(x(k+h+1), u(k+h), k+h) \\
& \text { s.t. } \forall h \in\{0, \ldots, H-1\} \\
& x(k+h+1)=A x(k+h)+B u(k+h) \\
& D_{d} u(k+h) \leq d \\
& e_{i}^{T} u(k+h) \leq \boldsymbol{\xi}_{i}(k+h), \quad \forall i=1, \ldots, n_{s} \\
& x(k+h+1) \in \mathcal{X}
\end{aligned}
$$

We make the following assumptions:

A.2. $j(\cdot)$ is a convex function with respect to $x$ and $u$.

A.3. At the first instant of the prediction horizon $k$, for all $i=1, \ldots, n_{s}$ the r.v. $\boldsymbol{\xi}_{i}(k)$ is known and for the rest of the prediction horizon $(h=1, \ldots, H-1)$, the marginal probability $\mathbb{P}\left(\boldsymbol{\xi}_{i}(k+h)=\xi_{n, i} \mid k\right)=\pi_{n, i}(k+h \mid k)$ is also known.

We highlight that problem (7) is unsolvable since we have yet to specify the meaning of the stochastic inequality $(7 \mathrm{~d})$. Based on the decision structure (Wets, 2002), different methods exist to solve such a problem.

Among them we focus on the Multistage Stochastic Programming which is used as contender to make a complexity and performance comparison with our proposal described in the section (4)

\subsection{Multistage Stochastic programming formulation}

The Multistage Stochastic programming(MSP) considers all the possible realizations of a r.v. at each stage to solve a unique optimization problem accordingly. The resulting

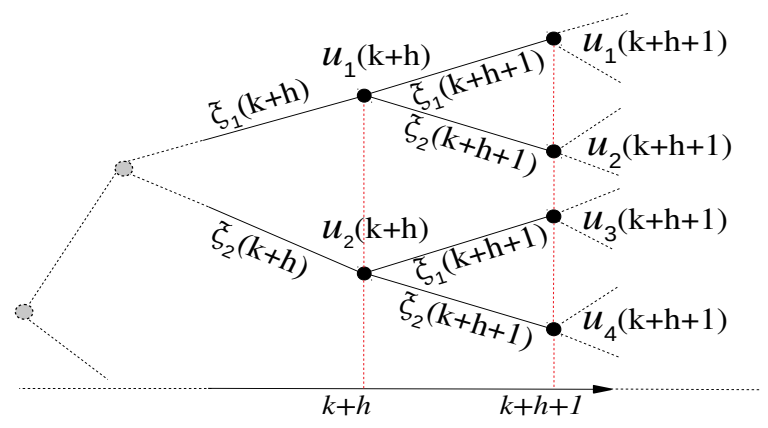

Fig. 1. MSP 
problem is a scenario tree that grows exponentially in consonance with the distribution of the r.v. and the prediction horizon. Each node of the tree at a particular stage corresponds to a realization of the r.v. at that stage and is associated with one or several decisions

Consequently to A.3., since the first decision of the sequence does not depend on r.vs. while the others do, the direct extension of problem (7) into the MSP framework is the "Wait and See" MSP. Figure 1 shows the scenario tree associated with a simple case of problem (7) where $n_{s}=1$, and $N_{1}=2$. The equivalent deterministic form of problem (7) in its MSP form is given by (8).

$$
\begin{aligned}
\min _{\mathbf{u}}\left\{j(x(k+1), u(k), k)+\sum_{s=1}^{S} \mathbb{P}(s)\right. \\
\\
\left.\sum_{h=1}^{H-1} j\left(x_{*, s}(k+h+1), u_{*, s}(k+h), k+h\right)\right\} \\
\text { s.t. } \forall s=1, \ldots, S ; \forall h \in\{0, \ldots, H-1\} \\
\quad x_{*, s}(k+h+1)=A x_{*, s}(k+h) \\
D_{d} u_{*, s}(k+h) \leq d \quad+B u_{*, s}(k+h) \\
x_{*, s}(k+h+1) \in \mathcal{X} \quad \\
\forall h \in\{1, \ldots, H-1\} \\
e_{i}^{T} u_{*, s}(k+h) \leq \xi_{*, s}(k+h)
\end{aligned}
$$

where $s, S$ and $\mathbb{P}(s)$ represent respectively a scenario (a particular sequence of the realization of the r.vs. over the prediction horizon), the total numbers of scenarios and the probability that the scenario $s$ occurs. The cost to be minimized is an expectation over all the possible scenarios.

\section{STOCHASTIC DISCRETE CONSTRAINT MODEL PREDICTIVE CONTROLLER (SDCMPC)}

We want to develop a controller that does not suffer from the combinatorial explosion of variables experienced by the MSP. That is, the decision $u$ over the prediction horizon does not depend on the realization of the random variable.

\subsection{Relaxed problem}

We propose to relax the constraints $(7 \mathrm{~d})$ using relaxation variables which, unlike $u$, can depend on the r.v. $\boldsymbol{\xi}_{i}(k+h)$. Our goal in doing so is to make the decision $u$ deterministic by transferring the associated randomness, to the relaxation variables. Therefore the relaxation variables become r.vs.. The relaxed constraint are given by:

$$
\begin{aligned}
\forall h=1, \ldots, H-1 ; \forall i=1, \ldots, n s & \\
e_{i}^{T} u(k+h)-\boldsymbol{y}_{i}(k+h) & \leq \boldsymbol{\xi}_{i}(k+h) \\
\boldsymbol{y}_{i}(k+h) & \geq 0
\end{aligned}
$$

Each relaxation variable $\boldsymbol{y}$ is random, because its optimal value is a function of its corresponding uncertain bound $\boldsymbol{\xi}$. It is often called recourse in the Stochastic Programming literature. We emphasize that there is no relaxation variable for the first instant $h=0$, since the random variable is supposed to be known already as a consequence of A.3.

The constraint violations allowed by the usage of the relaxation variables must be penalized, and hence a new term is added to the cost function of problem (7) such that the new cost function is:

$$
J_{S D C M P C}(k)=J(k)+J_{y}(k)
$$

with

$$
J_{y}(k)=\mathbb{E}\left[\sum_{h=1}^{H-1} \sum_{i=1}^{n_{s}} c_{i} \boldsymbol{y}_{i}(k+h)\right]
$$

where $c_{i}$ is the penalization associated to utilizing $\boldsymbol{y}_{i}$.

The control problem becomes a minimization on the decision variables $u$ and the recourse variables $\boldsymbol{y}$ function of $\boldsymbol{\xi}$. It can be solved by formulating its "deterministic equivalent".

\subsection{Deterministic equivalent}

We formulate the deterministic equivalent problem by introducing the variables $y_{1, i}(k+h), \ldots, y_{N_{i}-1, i}(k+h)$ all non negative which correspond to the various possible values taken by $\boldsymbol{y}_{i}(k+h)$ at the instant $k+h$. Therefore the stochastic constraints become a list of deterministic constraints as:

$$
\begin{aligned}
& \forall i=1, \ldots, n_{s}, \forall h=1, \ldots, H-1 \\
& \qquad\left\{\begin{aligned}
e_{i}^{T} u(k+h)-y_{1, i}(k+h) & \leq \xi_{1, i} \\
\vdots & \\
e_{i}^{T} u(k+h)-y_{N_{i}-1, i}(k+h) & \leq \xi_{N_{i}-1, i} \\
e_{i}^{T} u(k+h) & \leq \xi_{N_{i}, i}
\end{aligned}\right.
\end{aligned}
$$

We highlight that the last constraints ( $N_{i}$-th constraints) correspond to the higher value of the r.vs; hence, they describe hard constraints that should not be relaxed.

With this notation in mind and with A.3,

$$
\begin{aligned}
J_{\boldsymbol{y}}(k) & =\sum_{h=1}^{H-1} \sum_{i=1}^{n_{s}} c_{i} \mathbb{E}\left[\boldsymbol{y}_{i}(k+h)\right] \\
& =\sum_{h=1}^{H-1} \sum_{i=1}^{n_{s}} \sum_{n=1}^{N_{i}-1} c_{i} \pi_{n, i}(k+h \mid k) y_{n, i}(k+h) .
\end{aligned}
$$

The final problem to be solved is given by:

$$
\begin{aligned}
& \min _{u, y} J_{S D C M P C} \\
\text { s.t. } \forall h \in & \{0, \ldots, H-1\} \\
& x(k+h+1)=A x(k+h)+B u(k+h) \\
& D_{d} u(k+h) \leq d \\
& x(k+h+1) \in \mathcal{X} \\
\forall h \in & \{1, \ldots, H-1\}, \forall i=1, \ldots, n_{s} \\
& e_{i}^{T} u(k+h) \leq \xi_{N_{i}, i} \\
\forall i= & 1, \ldots, n_{s}, \forall n=1, \ldots, N_{i}-1 \\
& e_{i}^{T} u(k+h)-y_{n, i}(k+h) \leq \xi_{n, i} \\
& y_{n, i}(k+h) \geq 0
\end{aligned}
$$

Now that the formulation of the problem is finished lets us highlight some important points. 


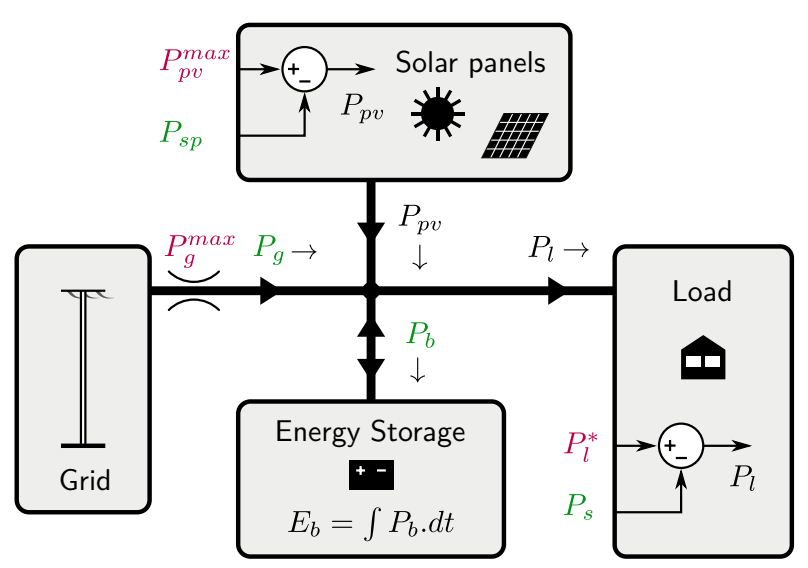

Fig. 2. Power flow model of a solar home. The decision variables are colored in green, external data are colored in red (Solar potential and desired consumption), internal variables are colored in black.

\subsection{Important remarks}

Let us consider problem (7). The total number of variables computed to solve this problem for the MSP is given by

$$
\left(n_{x}+n_{u}\right)\left[1+\sum_{h=1}^{H-1}\left(\prod_{i=1}^{n_{s}} N_{i}\right)^{h}\right]
$$

while it is

$$
n_{x}+n_{u}+(H-1)\left(n_{x}+n_{u}+\sum_{i=1}^{n_{s}} N_{i}-n_{s}\right)
$$

for the SDCMPC. A comparison between both numbers shows a great reduction from an exponential growth to a linear one with the new controller depending on the prediction horizon $H$. The difference in the number of variables is of great importance in time and memory usage performances, as we will show in section 6.4. Another advantage of the new formulation is that it is applicable for any type of r.vs. as long as the marginal probability of occurrence of the considered r.vs. $\pi_{n, i}(\cdot \mid k)$ can be computed.

It is also worth mentioning that $c_{i}$ introduced in (11) is a user setting parameter which exact value range is still an important open question. Depending on the chosen value, the control variables obtained by solving the optimization problem will be different, as we will show in the simulation and result section.

\section{ENERGY SYSTEM MODEL}

This section describes the different components of the energy model that is the study case shown in Figure 2. It is a model of a solar home, that is a grid-connected residential consumer equipped with photovoltaic power generation and an energy storage. We are interested in applying the strategy proposed in section 4 to this solar home, where there is an uncertainty about the electrical network availability. More details are given while describing the electrical network in the next section 5.1.

\subsection{System components}

The system has four main parts.

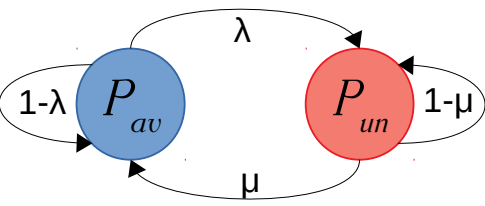

Fig. 3. Markov chain model of the electricity grid

The electrical network: The maximum power allowed to be drawn from the grid at each instant is given by a variable named $P_{g}^{\max }$ that can take either one of two values depending on whether the electrical network is available or unavailable. Thus $\boldsymbol{P}_{\boldsymbol{g}}^{\max }$ is a random r.v. which takes its values in $\left\{P_{a v}, P_{u n}\right\}$.

Explicitly, if the r.v.

- $\boldsymbol{P}_{\boldsymbol{g}}^{\boldsymbol{m a x}}=P_{u n}=0 \rightarrow$ the electricity network is not available, an outage is occurring;

- $\boldsymbol{P}_{\boldsymbol{g}}^{\boldsymbol{m a x}}=P_{a v} \rightarrow$ the electricity network is available, one can draw energy from it.

The maximum power allowed to be drawn from the electrical grid taking two values, either $P_{a v}$ or $P_{u n}$, we model the grid by a two states Markov Chain (MC) (Billinton and Allan, 1992) given by fig. 3 where $\lambda$ is said to be the failure rate while $\mu$ is the repair rate.

Let's $\boldsymbol{P}_{\boldsymbol{g}}^{\max }(k)$ denote the value of the electrical grid at time period $k$. Whenever the process is in some state, there is a fixed probability that it will be next in the other state or remain in the same. The one step transition probabilities matrix representing the $\mathrm{MC}$ is given by:

$$
\mathbf{T}=\left[\begin{array}{cc}
1-\lambda & \lambda \\
\mu & 1-\mu
\end{array}\right]
$$

Let's denote by $\Pi^{k+h}$ the h-step transition probability vector of the process from the initial instant $k$. The Chapman-Kolmogorov equations provide a method for computing the h-step transition probability vector such that:

$$
\begin{aligned}
\Pi^{k+h} & =\left[\pi_{a v}(k+h \mid k) \quad \pi_{u n}(k+h \mid k)\right] \\
& =\Pi^{k} \mathbf{T}^{h} \quad \forall h \geq 1
\end{aligned}
$$

Since the electricity grid state is supposed to be known at the initial instant (A.3.), the initial probability state vector $\Pi^{k}$ takes either the value $\left[\begin{array}{ll}1 & 0\end{array}\right]$ when the grid is available and $\left[\begin{array}{ll}0 & 1\end{array}\right]$ on the contrary.

We emphasize that this Markov chain is just a simple example, but more advanced reliability models could be used as well. In particular, our control method does not require the uncertain signals to be Markov processes.

The power needed to be drawn at each discrete time $k$ is the control variable $P_{g}$. Selling energy to the grid is not authorized, $P_{g}$ is non negative, therefore we have the following physical constraint:

$$
0 \leq P_{g}(k) \leq \boldsymbol{P}_{\boldsymbol{g}}^{\max } .
$$

The solar panel: Its production profile is $P_{p v}$. When the whole production $P_{p v}^{\max }$ defined by the solar irradiation cannot be utilized in the system, some part may be spilled. The power spilled $P_{s p}$ is a controlled variable such that the 
actual power provided by the solar panel to the system is given by:

$$
P_{p v}(k)=P_{p v}^{\max }(k)-P_{s p}(k)
$$

Energy storage: The decision variable $P_{b}$ (battery power) is the power sent to the battery at each instant. The accumulation of this power over time gives the energy within the storage $E_{b}$ (battery energy):

$$
E_{b}(k+1)=E_{b}(k)+P_{b}(k) \Delta_{t}
$$

with $\Delta_{t}$ the sampling time. By convention $P_{b}(k)>0$ and $P_{b}(k) \leq 0$ imply respectively charging and discharging.

Storage losses are neglected because they are out of scope here. The storage capacity at each instant is bounded as follows:

$$
E_{b}^{\min } \leq E_{b}(k) \leq E_{b}^{\max } .
$$

The load/building/user: The building demand at each instant $k$ is given by the variable $P_{l}^{*}$. When the building demand is above what the main (the electrical network) and the auxiliaries (the solar panel and the storage unit) sources can provide, some part of the demand must be shedded. The power to be shedded is the decision variable $P_{s}$. The actual power provided to the load $P_{l}$ can then be computed as follow:

$$
P_{l}(k)=P_{l}^{*}(k)-P_{s}(k) .
$$

We note here that introducing the decision variable $P_{s}$ into the problem permits to always satisfy A.1. (the control is always feasible).

These four previously described components exchange energy among themselves, which allows us to write the following load balance equation

$$
P_{g}(k)-P_{b}(k)+P_{p v}(k)=P_{l}(k)
$$

\subsection{System control objective}

The solar home controller aims to compute optimal decisions two satisfy two objectives:

(1) Minimizing the load dissatisfaction (maximizing the quality of service);

(2) Minimizing the economical cost (electricity bill);

both over the prediction horizon $\mathrm{H}$. We express the compromise between these two conflicting objectives through linear scalarization (Deb, 2014) which gives:

$$
\begin{aligned}
& j(x(k+1), u(k), k)=\underbrace{c_{g}(k) P_{g}(k)}_{\text {Economical cost }} \\
& +\underbrace{c_{s} P_{s}(k)}_{\text {Load dissatisfaction }},
\end{aligned}
$$

where $c_{g}$ is the time-varying energy price and $c_{s}$ a virtual price associated with one unit of discomfort. Both signals are known.

Since the load satisfaction objective is more important than the economical cost minimization, we set $c_{s} \gg$ $\max \left(c_{g}\right)$.

\section{SIMULATION AND RESULTS}

All of the simulations have been done using Julia (a highlevel, high-performance dynamic programming language for technical computing) with the Mosek solver, JuMP and "BenchmarkTools" (Revels, 2015) toolbox. The computer we have used run the Operating System Ubuntu 18.04.4 LTS and is powered by the processor Intel Core i7-8750H CPU @ $2.20 \mathrm{GHz}$ x 12 with $15.5 \mathrm{GiB}$ RAM.

\subsection{Simulation settings}

To simulate the controllers behavior, we use the the dataset extracted from the "Solar home electricity data" (Ratnam et al., 2017). The data-set comprises 3 years of data (solar power generated $P_{p v}^{\max }$ and household consumption $\left.P_{l}^{*}\right)$ recorded at a frequency of 30 minutes $\left(\Delta_{t}=0.5 \mathrm{~h}\right)$. In order to facilitate the interpretation of the results, let us define the net load power $P_{n l}$ as the difference between the building load demand and the solar panel total production.

$$
P_{n l}=P_{l}^{*}-P_{p v}^{\max }
$$

Note that $P_{n l} \geq 0$ means the local solar production is not enough to sustain the building's demand while the contrary $P_{n l}<0$ implies that there an excessive solar production that can be stored in the battery.

The electrical grid when available, provides a maximum energy $P_{a v}=3 \mathrm{kWh}$ (at a cost $0.1 €$ from 00:00 to $05 \mathrm{~h}: 59$ and $0.2 €$ from 06:00 to 23:59). The electrical distribution company also informs the subscriber of the failure and repair rate of the grid considered both constant. For the storage $E_{b}^{\max }=10 \mathrm{kWh}, E_{b}^{\min }=2 \mathrm{kWh}$ and we choose the shedding cost to be $c_{s}=10$.

\subsection{Manipulated variables}

For the problem considered, the manipulated variables are defined as follows:

- the system uncontrolled inputs are given by the a vector $v$ defined as

$$
v(k)=\left[\begin{array}{c}
P_{l}^{*}(k) \\
P_{p v}^{\max }(k)
\end{array}\right] ;
$$

- the decision or controlled variables are given by the vector $u$ defined as

$$
u(k+h \mid k)=\left[\begin{array}{c}
P_{g}(k+h \mid k) \\
P_{s p}(k+h \mid k) \\
P_{b}(k+h \mid k) \\
P_{s}(k+h \mid k)
\end{array}\right] ;
$$

- the stochastic variable is given by

$$
\boldsymbol{\xi}(k)=\boldsymbol{P}_{\boldsymbol{g}}^{\max }(k) .
$$

For our application there is a unique stochastic constraint which implies $n_{s}=1$ and therefore $i=1$. The stochastic variable takes its values within a set of two elements, meaning $N_{i}=N_{1}=2$ therefore, $n=1$. Given this, the elements present in the objective cost $J_{\boldsymbol{y}(k)}$ defined by equation (13) become $c_{i}=c_{1}, \pi_{n, i}(\cdot)=\pi_{1,1}(\cdot)=\pi_{u n}(\cdot)$ and $y_{n, i}(\cdot)=y_{1,1}(\cdot)$.

Note that the optimization variables is therefore given by

$$
\left[\begin{array}{c}
u(k+h \mid k) \\
y_{1,1}(k+h \mid k)
\end{array}\right]
$$




\subsection{Relaxation variable cost effect on the SDCMPC}

For the first set of simulations, we are interested in the influence of the parameter $c_{1}$ on the optimisation results. To do so, we solve problem (14) with a horizon of three days without any outage. We simulate four times with the varying parameter being $c_{1}$ (therefore $c_{1} \pi_{1,1}$ ) defined in the objective cost to be optimized given by (14a).

The results obtained from the simulation, are given on figures 4 and 5 . For clarity, we did not plot the relaxation variable $y_{1,1}$, because it is equal to $P_{g}$. We underline that drawing energy from the grid at the price $c_{g}(k+h)$ has the direct consequence of using the same quantity of relaxation variable at the price $c_{1} \pi_{1,1}(k+h \mid k)$. Table 1 presents a summary of the result obtained. In fig. 5, the dotted grey line represents the value of the shedding cost $c_{s}=10$.

Referring to figures 4,5 and table 1 we see that depending on the value of the product $c_{1} \pi_{1,1}(k+h \mid k)$ over the considered prediction horizon, we distinguish three types of behaviors of the controller:

- Behavior1: $c_{1} \pi_{1,1}$ is equal or higher than $c_{s}$. The controller sheds a part of demand instead of buying energy from the grid, the controller is in the over-preventive mode;

- Behavior2: $c_{1} \pi_{1,1}$ is bellow and far from $c_{s}$. The controller does not anticipate on the occurrence of outages, the controller is in the nominal mode;

- Behavior3: $c_{1} \pi_{1,1}$ is bellow but near $c_{s}$. The controller anticipate on the occurrence of a default on the grid, the controller is in the preventive mode .

From the three previously explained cases, we can assert that $c_{1}$ must always be chosen so that the controller never enters the over-preventive mode because not providing the building with its total energy demand when the grid is available is undesirable. Both remaining behaviors (nominal and preventive) can be selected by the user, depending on the cost $c_{1}$ and the MC parameters.

To choose the good value for $c_{1}$, let us recall that in the steady state of the MC described by Figure 3, the probability of being in state $P_{u n}$, is given by $\pi_{1,1}=\frac{\lambda}{\mu+\lambda}$. With this in mind the following equation is to be respected

$$
c_{1} \pi_{1,1}=c_{1} \frac{\lambda}{\mu+\lambda}=c_{s}^{\prime} .
$$

Note that $c_{s}^{\prime}$ is the maximum value we would like the cost $c_{1} \pi_{1,1}(k+h)$ to reach in steady state. Since $c_{1}$ depends on the MC parameters, it needs to be tune according to the user will every time these parameters change. For the rest of the paper, we would like the controller to be in the preventive mode. According to figure 5 the preventive

Table 1. SDCMPC Performances depending on the value of the parameter $c_{1}$

\begin{tabular}{lc|c|c} 
& $\sum P_{g} \Delta_{t}$ & $\sum C_{g} P_{g} \Delta_{t}$ & $\sum P_{s} \Delta_{t}$ \\
\hline$c_{1}=1$ & 19.27 & 1.97 & 0 \\
$c_{1}=10$ & 19.27 & 2.77 & 0 \\
$c_{1}=10^{2}$ & 6.46 & 0.65 & 12.8 \\
$c_{1}=10^{3}$ & 3 & 0.30 & 16.27 \\
\hline
\end{tabular}
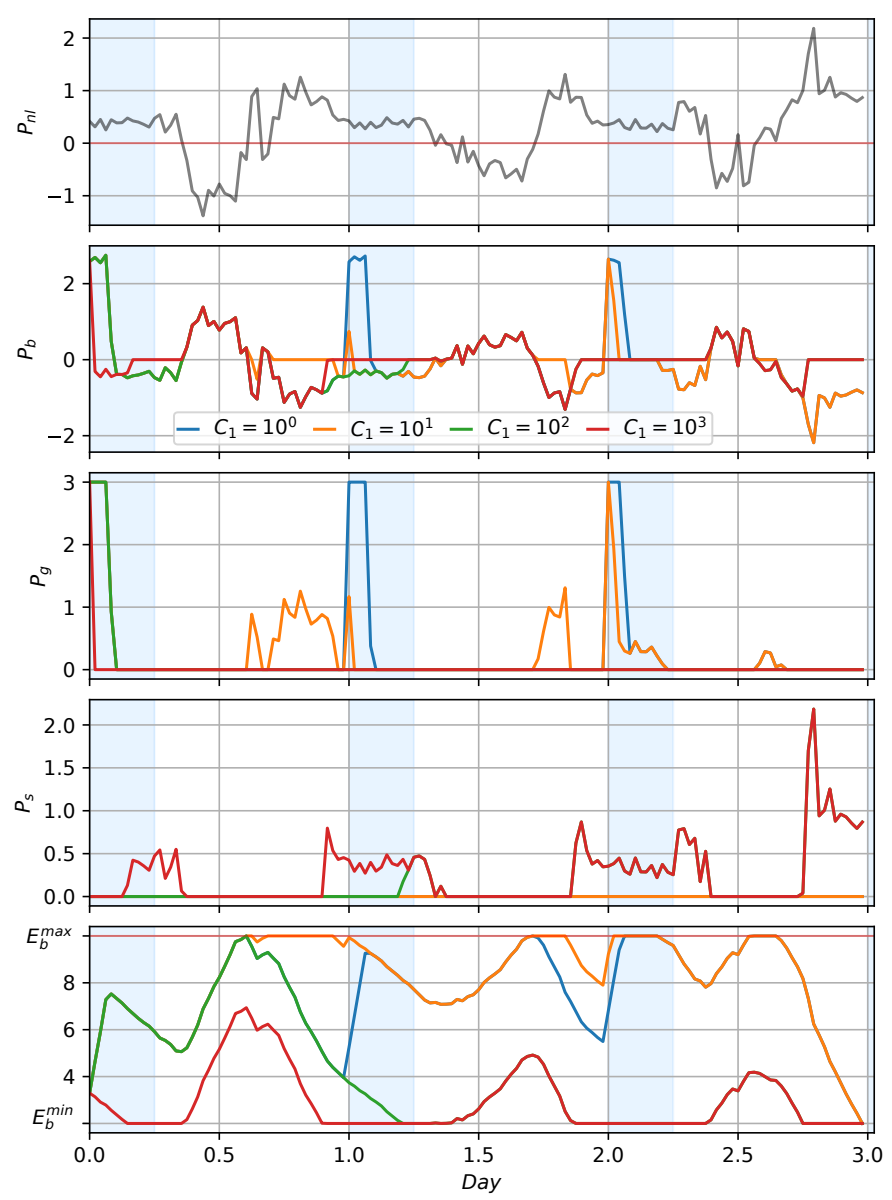

Fig. 4. Variation of power and state of charge depending on the cost $c_{1}$ associated to the relaxation variable for a constant failure and repair rate $\left(\lambda=10^{-2}, \mu=10^{-2}\right)$ of the grid. The parts colored in light black correspond to the moment of the day where the electricity price is the lowest

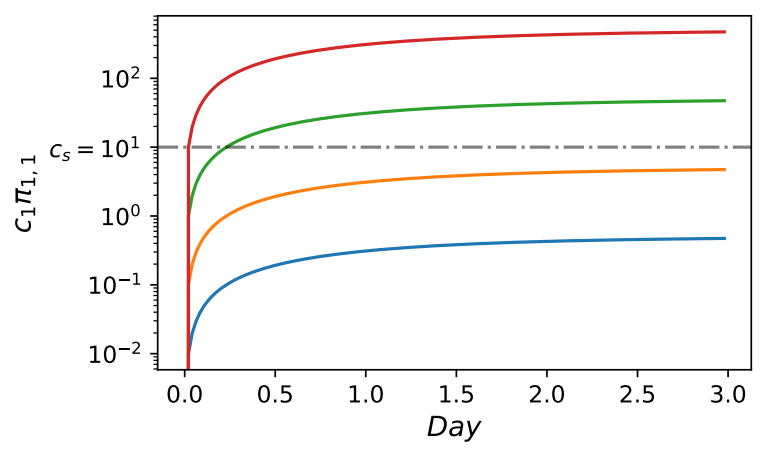

Fig. 5. Variation of the product $c_{1} \pi_{1,1}(k+h)$ associated to the relaxation variable for a constant failure and repair rate of the grid.

mode is activated for a $c_{s}^{\prime} \approx 5$ which leads to $c_{1}=10$ for the following simulations.

\subsection{Performances comparison}

For the optimisation problem, we are interested in comparing the computing time and memory usage of the SDCMPC and the state of the art controller MSP (described 
Table 2. Stochastic Controller Performances for a Monte Carlo simulation of 10 days in Closed loop with a prediction horizon of 7.5 hours for SDCMPC and MSP and 24 hours for SDCMPC2

\begin{tabular}{lrr||ccc||cc}
\cline { 2 - 6 } & \multicolumn{2}{c}{$\sum j_{r e}$} & \multicolumn{2}{c}{$\sum C_{g} P_{g} \Delta_{t}$} & \multicolumn{2}{c}{$\sum P_{s} \Delta_{t}$} \\
\cline { 2 - 7 } & Mean & Std & Mean & Std & Mean & Std \\
\hline MSP - SDCMPC & 36.75 & 22.54 & -1.03 & 0.47 & 3.78 & 2.29 \\
SDCMPC - SDCMPC2 & 58.15 & 40.24 & 0.74 & 1.22 & 5.74 & 4.11 \\
MSP - SDCMPC2 & 94.9 & 48.88 & -0.29 & 1.21 & 9.52 & 4.98 \\
\hline
\end{tabular}
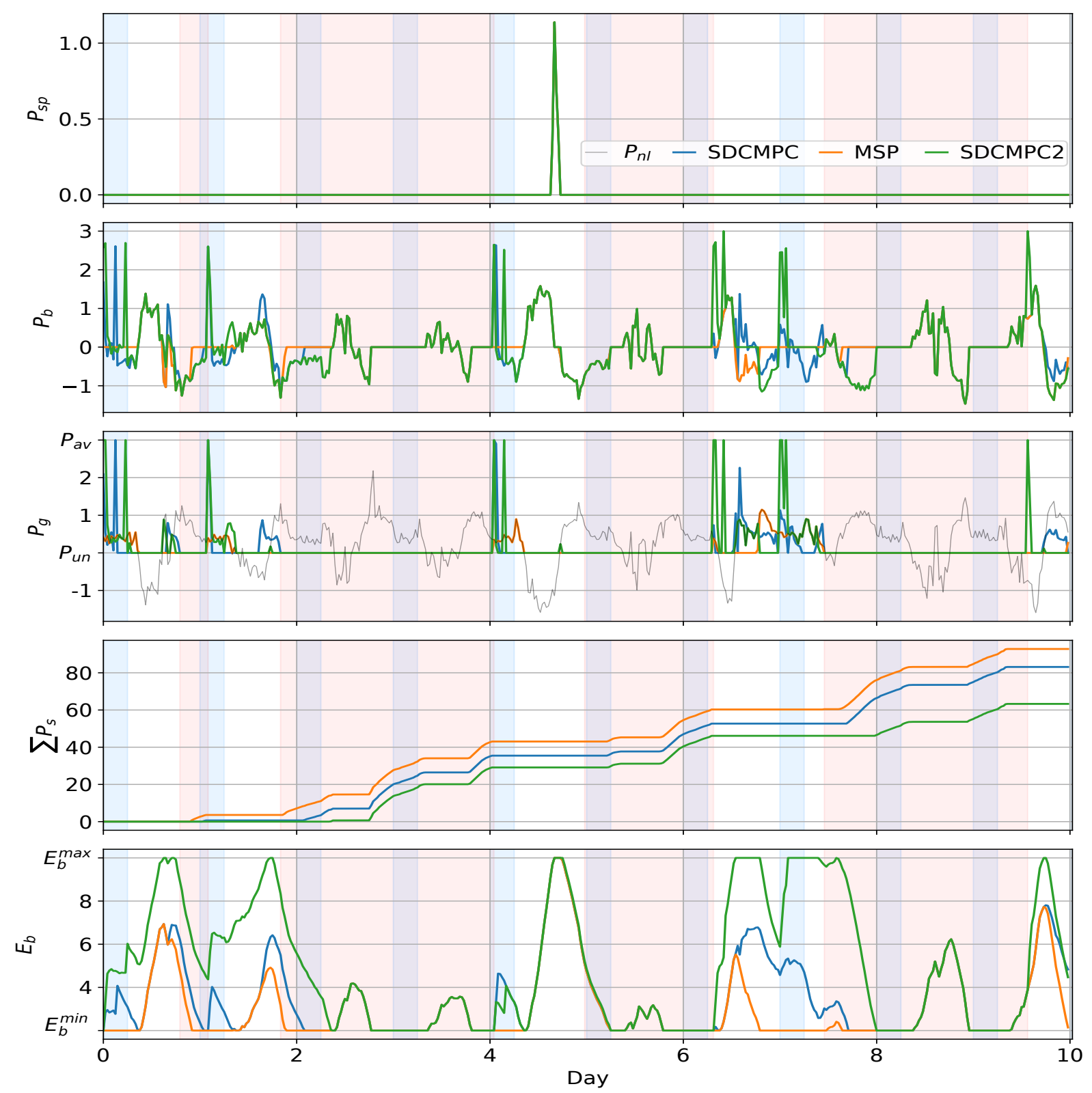

Fig. 6. Evolution of the variables associated with SDCMPC, MSP (prediction horizon $=7.5$ hours for both) and SDCMPC2 (prediction horizon $=24$ hours) of scenario 24 .

in section 3.2) for different values of the prediction horizon $\mathrm{H}$.

The results obtained with these simulations are shown in figure 7 . We can see that the memory usage and the computing time both grow exponentially according to the prediction horizon for the MSP, while the growth is much much slower for the SDCMPC. Let emphasize that the laptop ran out of memory for a prediction horizon higher than 7.5 hours with the MSP. The conclusion is pretty straightforward; for the same prediction horizon, the SDCMPC has better computing time and memory usage than the MSP. The low computing time and memory usage of the SDCMPC allows extending its prediction 

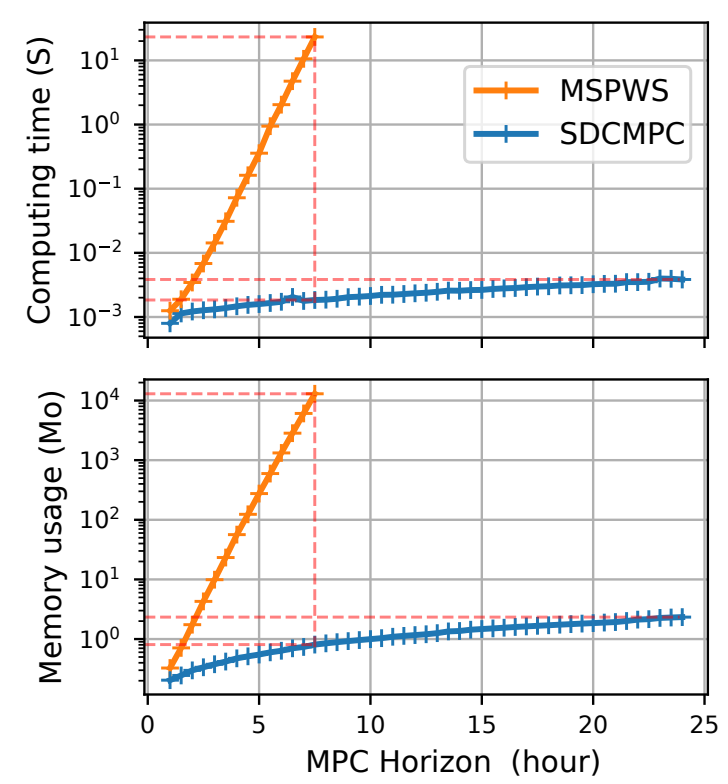

Fig. 7. Evolution of the computing time and memory estimate usage function of the prediction horizon from 1 to 7.5 hour for the MSP and from 1 hour to 24 hour for the SDCMPC

horizon from the maximum admissible by the MSP to a much longer one. For this paper, we consider a whole day (24 hours) as the longest prediction horizon. Figure 7 shows that even at the prediction horizon of 24 hours for the SDCMPC, there still a considerable difference in computing time and memory usage compared to the MSP.

\subsection{Monte Carlo simulation}

Based on a failure rate $\lambda=10^{-2}$ and a repair rate $\mu=$ $10^{-2}$ for the Markov chain describing the grid state, we have created 40 independent scenarios of grid availability over ten days. All scenarios share the same profile of house demand and solar production. We have simulated the SDCMPC and MSP with a prediction horizon of 7.5 hours and the SDCMPC considering the extended horizon 24 hours (SDCMPC2). Figure 6 shows the evolution of the interesting variables for one of these scenarios. Translucent red is used to highlight grid outages while black is used for low-cost electricity during the night. The fourth subplot represents at each instant the integral over time of energy not provided to the house $P_{s} . P_{n l}$ ("net load") is the difference between $P_{l}^{*}$ and $P_{p v}^{\max }$.

For each scenario and each controller, we have computed the following performance indices:

- Cumulated cost of energy drawn from the grid (energy bill)

- Cumulated energy not provided to the building

- Cumulated reconstructed optimization cost (weighted sum of the first two $): j_{r e}(k)=j(x(k+1), u(k), k)$

There is a large variability across the 40 scenarios (grid availability is $53 \%$ on average for all scenarios, but it is only $3 \%$ in the worst one and $98 \%$ in the luckiest). Therefore, comparing the average performance across all scenarios for each controller yields statistically meaningless differences. Instead, for each scenario, we compute
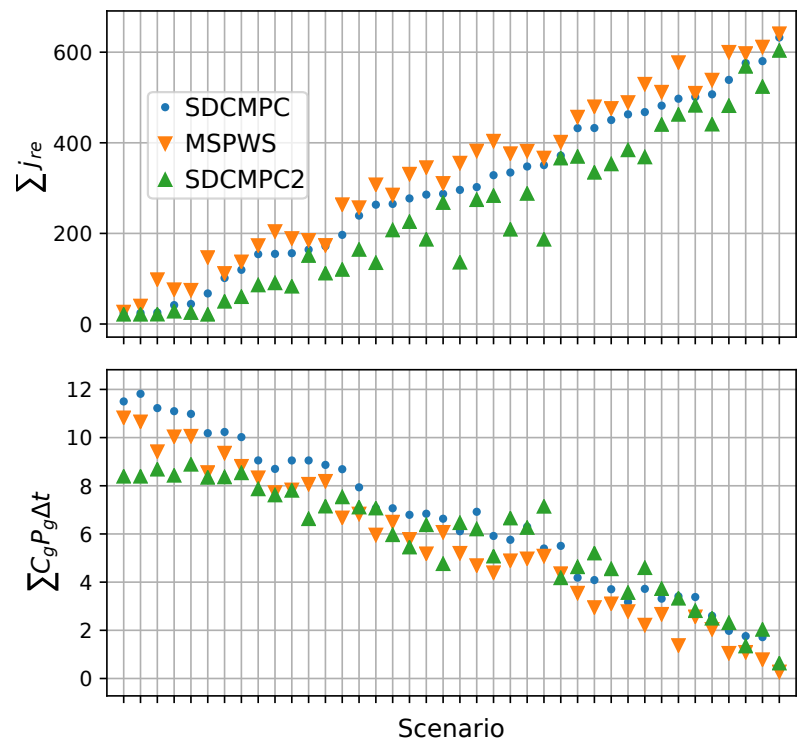

Fig. 8. Reconstructed optimization cost rearranged in ascending order of the results obtained by the OLFC with an horizon of 7.5 hour

differences in performance indices for pairs of controllers. In table 2, we show the mean and the standard deviation of these differences across all scenarios. The SDCMPC2 does better on average compared to other controllers. This is due to its higher prediction horizon, which allows better anticipation, in particular, to take most of grid energy at a lower price.

Figure 8, we show the performance indices for each scenario (dropping the shedded energy, because it is very similar to the total reconstructed cost $\sum j_{r e}$ due to the high $c_{s}$ weight) and each controller. For plotting clarity, the scenarios are sorted in ascending order of the cummulated reconstructed optimization cost $\left(\sum j_{r e}\right)$ for SDCMPC. This yields an approximate ascending order for the other controllers' cost and approximate descending order for the energy bill. Indeed, the energy bill is negatively correlated with grid unavailability and, therefore, the energy dissatisfaction.

We can see that the SDCMPC2 yields the lowest $j_{r e}$ for all scenarios, followed by the MSP, and then the SDCMPC. As for the energy bill, the SDCMPC2 can be lower, higher, or equal to the other controllers depending on the scenario. However, even for scenarios where SDCMPC2 energy cost is worse (higher) than the other's, this is more than compensated by a lower energy dissatisfaction.

\section{CONCLUSION}

We have proposed in this paper a controller that integrates discrete stochastic constraints in the MPC framework. To do so, we have used relaxation techniques within an Open Loop Feedback Control formulation. The resulting optimization complexity is not much increased compared to a deterministic MPC. On an energy management application, we have compared the new controller to a controller based on the MSP. Our controller's very low computational time and memory usage compared to the MSP allows a substantial extension of its prediction horizon. The ex- 
tended horizon results in a significant improvement in the quality of service and the closed-loop optimization cost.

This approach is very well suited for the linear discrete stochastic constraints that model the commutations in this work. Further works need firstly to study its adaptation to other aspects of hybrid dynamic systems. Lastly, it would be interesting to reevaluate the MSP while assuming a deterministic trajectory of the random variable after a predetermined reduced horizon or by cutting the less likely scenarios in the tree.

\section{REFERENCES}

Adam, L., Branda, M., Heitsch, H., and Henrion, R. (2018). Solving joint chance constrained problems using regularization and benders' decomposition. Annals of Operations Research.

Bemporad, A. and Morari, M. (1999). Robust model predictive control: A survey. In A. Garulli and A. Tesi (eds.), Robustness in identification and control, 207-226. Springer London, London.

Ben-Tal, A., Ghaoui, L.E., and Nemirovski, A. (2009). Robust Optimization. Princeton Series in APPLIED MATHEMATICS.

Bertsekas, D.P. (2005). Dynamic programming and suboptimal control: A survey from adp to mpc. European Journal of Control, 11(4-5), 310-334. doi:10.3166/ejc. 11.310-334.

Billinton, R. and Allan, R.N. (1992). Reliability Evaluation of Engineering Systems. Springer US.

Borrelli, F. (2017). Predictive Control for Linear and Hybrid Systems. Cambridge University Press, 1rst edition.

Bram, L., Yamkog̃lu, I., and den Hertog, D. (2015). A practical guide to robust optimization. Omega, 53, 124 $-137$.

Camacho, E.F. and Alba, C.B. (eds.) (2007). Model Predictive Control. Springer-Verlag London, 2nd edition.

Charnes, A., Cooper, W.W., and Symonds, G.H. (1958). Cost horizons and certainty equivalents: An approach to stochastic programming of heating oil. Management Science, 4(3), 235-263.

Deb, K. (2014). Multi-objective Optimization, 403-449. Springer US, Boston, MA.

Dreyfus, S.E. (1964). Some types of optimal control of stochastic systems. Journal of the Society for Industrial and Applied Mathematics Series A Control, vol.2(No. 1), $120-134$.

Geng, X. and Xie, L. (2019). Data-driven decision making with probabilistic guarantees (part 1): A schematic overview of chance-constrained optimization. arXiv: Optimization and Control. Review on chance constrained programing methodes.

Henrion, R. (2007). Structural properties of linear probabilistic constraintst. Optimization, 56, 425-440. doi: 10.1080/02331930701421046.

James B. Rawlings, David Q. Mayne, M.M.D. (2017). Model Predictive Control: Theory, Computation, and Design, 2nd Edition. Nob Hill Publishing, LLC.

Lucia, S., Andersson, J.A., Brandt, H., Diehl, M., and Engell, S. (2014). Handling uncertainty in economic nonlinear model predictive control: A comparative case study. Journal of Process Control, 24(8), 1247 - 1259.

Mayne, D. (2016). Robust and stochastic model predictive control: Are we going in the right direction? Annual Re- views in Control, 41, 184-192. doi:10.1016/j.arcontrol. 2016.04.006.

Mayne, D.Q. (2014). Model predictive control: Recent developments and future promise. Automatica, 50(12), 2967-2986. doi:https://doi.org/10.1016/j.automatica. 2014.10.128.

Nasir, H.A., Care, A., and Weyer, E. (2019). A scenariobased stochastic mpc approach for problems with normal and rare operations with an application to rivers. IEEE Transactions on Control Systems Technology, 27(4), 1397-1410.

Pacaud, F., Carpentier, P., Chancelier, J.P., and De Lara, M. (2018). Stochastic optimal control of a domestic microgrid equipped with solar panel and battery.

Papageorgiou, M. (1988). Certainty equivalent open-loop feedback control applied to multireservoir networks. IEEE Transactions on Automatic Control, 33(4), 392399.

Pereira, M.V.F. and Pinto, L.M.V.G. (1991). Multistage stochastic optimization applied to energy planning. Mathematical Programming, 52(1), 359-375.

Prékopa, A. (1970). On probabilistic constrained programming. In Proceedings of the Princeton Symposium on Mathematical Programming, 113-138. Princeton University Press.

Prince Agbodjan, J.J., Haessig, P., Bourdais, R., and Gueguen, H. (2019). Resilience in energy management system: A study case. IFAC-PapersOnLine, 52(4), 395 - 400. IFAC Workshop on Control of Smart Grid and Renewable Energy Systems CSGRES 2019.

Prince Agbodjan, J.J., Haessig, P., Bourdais, R., and Gueguen, H. (2020). Stochastic modelled grid outage effect on home energy management. 2020 4th IEEE Conference on Control technology and Application (CCTA).

Raković, S. (2015). Robust Model-Predictive Control, 1225-1233. Springer London, London. doi:10.1007/ 978-1-4471-5058-9_2.

Ratnam, E.L., Weller, S.R., Kellett, C.M., and Murray, A.T. (2017). Residential load and rooftop PV generation: an Australian distribution network dataset. International Journal of Sustainable Energy, 36(8), 787-806.

Revels, J. (2015). Benchmarktools. https ://github.com/ JuliaCI/BenchmarkTools.jl. Accessed: 2020-06-18.

Wets, R.J.B. (2002). Stochastic programming models: Wait-and-see versus here-and-now. In C. Greengard and A. Ruszczynski (eds.), Decision Making Under Uncertainty, 1-15. Springer New York, New York, NY. 